\title{
Integrative Concepts for Liver Surgery
}

\author{
Stefan Heinrich Felix Watzka Hauke Lang \\ General, Visceral and Transplantation Surgery, University Hospital, Mainz, Germany
}

\section{Keywords}

Liver resection - Associating liver partition and portal vein ligation · Two-stage concept · Liver cancer · Liver metastases

\begin{abstract}
Background: Surgery is the standard treatment for primary tumors and metastases. Due to improvements in surgical outcomes as well as the efficacy of systemic treatments, the role of surgery has changed in recent years. Summary: Liver surgery has become safe and efficient, with resectability being increased by multimodality concepts as well as staged liver resections and orthotopic liver transplantation. These concepts may be applied to primary liver tumors but also to selected patients with liver metastases from various diseases. In addition, even debulking surgery may be indicated for selected patients with endocrine metastases. While patient selection for liver resections was limited to clinical parameters in the past, histological and molecular characteristics have become increasingly important. Moreover, the response to regional or systemic chemotherapy has been demonstrated to be strong for a beneficial course of the disease even in advanced diseases. Key-Messages: Due to the variety of available treatment options, optimal patient selection is crucial. Besides liver surgery, staged concepts as well as liver transplantation are curative tools for many patients.
\end{abstract}

(c) 2020 S. Karger AG, Basel

\section{Introduction}

Surgery has become the standard treatment for primary and many secondary liver tumors. Precision medicine may refer to tailored treatments to a particular disease, biological tumor characteristics, and the individual patient. For liver tumors, such tailored treatment concepts usually include the choice of the optimal surgical strategy and perioperative management with regard to tumor-directed local or systemic treatments. Due to the different biologic behaviors and underlying diseases as well as the varying availability of potent systemic treatment options, these concepts vary between the different entities.

The most frequent malignant primary liver tumors are intrahepatic cholangiocellular (iCCC) and hepatocellular carcinoma (HCC). However, the liver is even more often affected by metastases from different primaries, of which colorectal cancer (CRC) is by far the most frequent primary tumor.

\section{Primary Liver Tumors}

\section{Hepatocellular Carcinoma}

Recent publications suggest a rationale for anatomical (e.g., segmental) resections in HCC in contrast to iCCC and liver metastases, since this tumor type tends to spread locally within segmental compartments [1]. While extremely rare in resectable HCC in cirrhosis, HCC in noncirrhotic liver parenchyma are sometimes associated with hilar lymph node (LN) metastases. In particular, fibrola- 
mellar HCC (FLC) has shown a high risk of hilar LN metastases (35-50\%) in some series [2-4]. Given the low morbidity of a lymphadenectomy in noncirrhotic patients, a hilar lymphadenectomy should therefore be performed whenever an FLC is suspected and it should be taken into consideration for all patients with HCC in noncirrhotic livers. In contrast, a hilar lymphadenectomy should be considered very carefully in patients with liver cirrhosis given a higher morbidity in portal hypertension in these patients and the generally low level of evidence.

In noncirrhotic livers, even extended resections are feasible, and liver resection is the first-line treatment. However, HCC predominantly arises from diseased liver parenchyma, mainly liver cirrhosis. Therefore, orthotopic liver transplantation (OLT) has become an attractive treatment option for HCC since surgery in liver cirrhosis is associated with a higher risk of liver failure and the trigger for HCC development remains after liver resection in a cirrhotic liver. OLT not only removes the HCC but also cures the underlying disease and normalizes liver function.

However, OLT requires life-long immunosuppression, which, on the other hand, increases the risk of infectious as well as secondary benign (hypertension, diabetes, etc.) and malignant diseases (skin cancer, lymphoma, etc.) in the long term. Depending on the tumor characteristics, immunosuppression might also increase the risk for HCC recurrence after OLT. Overall and disease-free survivals are not impaired in patients with an HCC meeting the Milan criteria (solitary HCC up to $5 \mathrm{~cm}$ and 3 HCC up to $3 \mathrm{~cm}$ each) after OLT compared to patients transplanted without HCC [5].

The main histological determinant for a high recurrence risk is the presence of microvascular invasion (MVI) [6]. In addition, a novel parameter might be the vessel encapsulating tumor cluster, which is also associated with a higher recurrence rate after surgical therapy [7]. Since both features are difficult or even impossible to assess on preoperative biopsies, these biological parameters are not included in the preoperative treatment planning for HCC. They rather serve as prognostic factor postoperatively. Consequently, current guidelines for the management of HCC recommend resection in patients with a preserved liver function, while OLT is mainly offered to patients with impaired liver function [8]. In case of disease recurrence, those patients who primarily underwent liver resection or ablation are usually evaluated for "salvage" OLT [9]. However, since retrospective analyses suggest a survival benefit after primary OLT compared to resection followed by subsequent OLT, many patients with (small) HCC in compensated liver cirrhosis are already initially considered as candidates for OLT [ 10 , 11]. Although OLT primarily appears to be more invasive than liver surgery, morbidity of liver surgery in cirrhosis is substantial, and many patients do not qualify for OLT in case of disease recurrence anymore [10].

More problematic are patients with HCC outside of the Milan criteria. While those with a preserved liver function (e.g., Child A) may be considered for liver surgery, patients with impaired liver function are not candidates for resection surgery. According to a consensus conference on HCC, the eligibility criteria for OLT should be expanded for these patients in order to offer a potentially curative OLT also to these patients, since the overall survival of those patients after OLT is by far better than after palliative treatment [12].

Moreover, the literature suggests a favorable outcome of patients with HCC outside of the Milan criteria without MVI [6]. Since MVI cannot be assessed preoperatively, other "biological" selection criteria have been evaluated, and the response to neoadjuvant transarterial chemoembolization (TACE) appears very promising; patients with HCC outside the Milan-criteria who responded to TACE revealed tumor-specific survival after OLT comparable to HCC within the Milan-criteria $[13,14]$. Also, stable disease or the response to TACE of patients with HCC within the Milan-criteria indicates a favorable outcome, while disease progression within this group of patients is associated with significantly worse outcomes [14]. Up to now, this selection criterion has not increased the priority in the allocation process.

\section{Intrahepatic Cholangiocellular Carcinoma}

Surgery used to be the only treatment with curative potential for patients with iCCC. In contrast to HCC, intrahepatic CCC mainly arises from noncirrhotic parenchyma, and the diagnosis is often made in an advanced disease stage. The surgical strategy aims at $\mathrm{R} 0$ resection without a defined resection margin. The rate of $\mathrm{LN}$ metastases is comparably high in iCCC, and therefore extension of the hilar lymphadenectomy to LN at the lesser gastric curve or behind the pancreas is recommended in iCCC in the left or right liver lobe, respectively.

In addition to distant metastases, established risk factors for tumor recurrence are multifocal disease, tumor size, LN metastases, incomplete resection (R1), and infiltration of surrounding tissue. While surgery for these often advanced tumors can be performed safely with acceptable morbidity and mortality, tumor recurrence is frequent and recurrence-free survival short. The preoperative prediction (POP) score accurately stratifies the recurrence risk prior to surgery based on imaging criteria of the intrahepatic disease load into 3 risk groups; all patients in the high-risk group developed recurrence within 1 year of surgery. In contrast, a substantial proportion of patients did not develop recurrence during the follow-up period at all in the low-risk group [15]. According to these data, patients in the high-risk group should be excluded from sur- 
gery in the absence of a potent systemic treatment. Neoadjuvant therapy may be the key for these patients, since they might be considered surgical candidates in case of a tumor response to neoadjuvant chemotherapy. However, such concepts require a prospective evaluation. Similarly, future studies need to assess for different risk-groups whether a neoadjuvant or perioperative concept would be superior to the adjuvant concept, which is currently considered standard of care in resected iCCC [16].

\section{Liver Metastases}

\section{Colorectal Liver Metastases}

The liver is the only site of metastasis in the majority of patients with CRC, and liver surgery has developed into a cornerstone in the management of CRC liver metastases (CRLM) in recent decades. Metastatic disease is stratified as primarily resectable, borderline resectable, or unresectable. However, clear definitions for these terms do not exist, and the evaluation of resectability varies even among experts [17].

Due to the proven curative potential of liver surgery, the goal of concepts for all 3 groups of CRLM metastases is the complete removal of metastases. Often, surgery is part of a multimodality treatment concept that may aim to achieving resectability by downsizing metastases or preventing recurrence. Resectability is limited by the functional capacity of the future liver remnant (FLR), which is the remnant parenchyma after complete resection of all metastases. Depending on the function and quality of the parenchyma, at least $25 \%$ of the liver volume with adequate in- and outflow needs to be preserved. Intense and long chemotherapy is well known to impair liver function, which limits the extent of liver surgery and increases the minimal volume of the FLR [18].

\section{Two-Stage Concepts}

One of the classical surgical concepts to achieve resectability is 2 -stage resection. The liver has the unique potential to regenerate its volume after major liver resections, and this potential can be used for repetitive or staged resections of bilateral metastases. Also, repetitive resections of recurrent liver metastases are technically feasible if sufficient liver parenchyma with adequate arterial and portal venous perfusion as well as venous drainage remains. Second, third, and fourth resections have been described. The oncological outcome is comparable to that of primary liver resections with a low morbidity and mortality [19]. In the own experience, repeat resections up to 6 resections have been feasible in selected patients.

Bilateral metastases can be resected by 2 -stage concepts. In step 1 metastases of one side (usually left) are removed by atypical resections. After sufficient hypertrophy, a formal or extended hemihepatectomy can be performed safely to remove metastases in the right hemi-liver. In order to further increase the volume of the left liver segments prior to a hemihepatectomy, the portal vein to the right side can be ligated during the first step of the operation (PVL) or embolized (PVE) after the first step [20]. Hypertrophy is usually maximal after 4-6 weeks. However, in patients with a high recurrence risk, the second step may also be postponed for several months and a systemic chemotherapy administered between steps 1 and 2 .

These concepts may also be used in synchronous metastases, when resection of the primary tumor is combined with clearance of one side of the liver, and the contralateral lobe is resected after a certain period of systemic chemotherapy.

Recently, the PVE concept has been extended by additional (simultaneous or sequential) embolization of the right hepatic vein [21]. This combination results in more rapid and intense hypertrophy of the contralateral lobe than PVE alone.

The fastest hypertrophy has been reported for the associating liver partition and portal vein ligation (ALPPS), which combines a complete (or at least $50 \%$ partial) transection of the liver parenchyma between segment 4 and the left lateral sector and ligation of all portal venous branches to the right liver during the first step [22]. Atypical resections in segments 2 and 3 may even be added. An extended right hemihepatectomy is performed about 7-10 days later, when the FLR has usually hypertrophied to a sufficient liver volume. Up to now, the speed of liver regeneration in response to the ALPPS is not fully understood [23]. This procedure is technically demanding and has a substantial morbidity and mortality rate. In particular, complications during the 2 steps have detrimental effects $[22,24]$.

These staged concepts help to increase the resectability of liver metastases. Although liver regeneration in general has been suspected for a long time to trigger tumor growth, such an effect has not been confirmed for the ALPPS concept [25]. Moreover, the first randomized trial on ALPPS versus the conventional 2-stage concept demonstrated a better recurrence-free and overall survival after ALPPS [26]. Of note, patients were required to have at least stable disease after 3 months of systemic chemotherapy prior to study inclusion, which reflects a positive patient selection.

In the own institution, bilateral metastases are resected during one operation, whenever possible. If the FLR appears too small after clearance of all metastases, a staged concept is performed. The choice of PVL or PVE is mainly dependent on anatomical considerations. We only use the ALPPS approach if PVE/PVL have failed or the FLR appears very little [27]. 
Parenchyma-Sparing Resection Concept

Surgery for CRLM has changed over time; initially anatomical resections were the preferred type of resection with margins of $1 \mathrm{~cm}$ or at least $0.5 \mathrm{~cm}$. Currently, R0 resection of any tumor-free margin is the goal of any liver resection for CRLM $[28,29]$. In case of a tumor response to preoperative chemotherapy, even $\mathrm{R} 1$ resections seem to be adequate since they have recurrence rates comparable to those of R0 resections. Moreover, $\mathrm{R} 1$ resection at the site of tumor contact to major blood vessels (glissonian trias or hepatic veins) presumably does not increase the recurrence risk at all [30]. In contrast, the classical approach aimed at a circumferential $\mathrm{R} 0$ resection requiring the resection of the affected vessel and by this often major anatomical resections.

In contrast, reports increasingly suggest that metastases from K-ras mutant tumors more often contain peritumoral micrometastases, which results in smaller resection margins. Therefore, wider resection margins and anatomical resections are under debate for K-ras-mutant liver metastases [31-33].

\section{Orthotopic Liver Transplantation}

With the concept of a "localized" rather than a "systemic" disease, also OLT appears attractive for CRLM, since OLT guarantees 100\% resectability of liver metastases and hepatic micrometastases. However, early reports on OLT for CRLM revealed poor long-term survival due to early recurrence associated with immunosuppression [34]. Therefore, CRLM had been considered a strict contraindication for OLT in the past.

Just recently, a prospective trial demonstrated excellent long-term survival in patients with unresectable CRLM who underwent OLT [35]. Patients with a tumor response to systemic chemotherapy of $10-30 \%$ depending on the extent of metastasis or a response to TACE of at least $20 \%$. Moreover, the diagnosis of the CRC primary had to be at least 1 year before the listing for OLT. Before chemotherapy, the largest metastasis had to be below $10 \mathrm{~cm}$. Recurrence of metastasis was not an exclusion criterion, but chemotherapy had to be the firstline treatment [36]. In this selected cohort of patients, the metabolic tumor volume measured from pre-OLT positron-emission tomography was highly predictive of long-term survival [37]. Patients who did not meet these selection criteria revealed a dismal outcome after OLT [38]. The rationale for selection by chemotherapy is mainly the concept of micrometastases, which may exhibit accelerated tumor growth under immunosuppression.

Moreover, OLT with consequent immunosuppression seems not to impair the quality of life in the long term: although the general health status, physical function, and role function scores were significantly lower 3 months af- ter OLT compared to pre-OLT values, they were comparable to baseline scores again 6 months after OLT again [39].

\section{Recurrence Prediction}

Most importantly, the potential gain of prognosis due to resection has to be weighed against the potential morbidity of a surgical concept in each particular patient. Clinical risk scores for recurrence-free and overall survival have been developed, which are mainly based on the timing of metastasis, involvement of LN of the primary tumor and the level of the tumor marker CEA [40]. These scoring systems are highly predictive of tumor recurrence, of which the Basington predictive index appears to be the most accurate. Accordingly, extensive surgery may be offered to a young and healthy patient despite a highrisk score in the Basington predictive index, while older patients with comorbid diseases may only be offered a resection for less extensive, e.g., metachronous, metastases of a node-negative primary tumor (low-risk) in weighing of the prognosis and surgical outcome [40].

As for chemotherapy, localization of the primary tumor (right vs. left colon) is also prognostic after liver surgery, with right-sided tumors having a worse prognosis [41]. K-ras mutations occur more frequently in right-sided CRC (40 vs. $20 \%$ ) and are associated with impaired survival in CRLM. While OS is shorter in right-sided Kras wild-type tumors, the localization has no effect on OS in K-ras mutant tumors [41]. In addition to K-ras, other genetic parameters such as microsatellite instability, BRAF mutations, and combinations of aberrations in tumor suppressor genes have been demonstrated to be predictive of outcomes after liver resection [42]. In the own experience, the inclusion of mutations in smad family genes into a clinical score was highly predictive of outcomes after liver resection of CRLM [43].

\section{Patient Selection by Chemotherapy}

As indicated above, a multimodality treatment is often initiated for CRLM. Since the tumor response is associated with prognostic markers of CRC (e.g., K-ras status), the radiological response to neoadjuvant or downsizing chemotherapy is also associated with a beneficial outcome after liver surgery. Accordingly, the pathological tumor response is one of the strongest predictors of outcome after CRLM liver surgery [44].

Moreover, patients with initially unresectable liver metastases reveal a prognosis comparable to that of patients with primarily resectable metastases after efficient downsizing and secondary resection $[45,46]$. Vice versa, tumor progression during chemotherapy is an independent risk factor for poor outcomes even if a $\mathrm{R} 0$ resection has been achieved [47]. Accordingly, the response to chemotherapy was a major selection criterion in the first ran- 
domized trial on ALPPS (SECA-1 study) which revealed impressive long-term survival after OLT for unresectable CRLM [35].

\section{Liver Metastases of Neuroendocrine Neoplasms}

Compared to CRC, neuroendocrine neoplasms (NEN) are a very rare and an extremely heterogeneous group of tumors. They may be associated with clinical symptoms caused by their hormonal activity. Although the majority of NEN show no hormonal activity in the early period of the disease (85\%), a clinical endocrine function often occurs with the development of liver metastases. Around $12-34 \%$ of patients present with distant metastases at the initial diagnosis, and liver metastases (NELM) occur in $50-95 \%$ of cases during the further course of the disease and determine the prognosis and the quality of life of the patient [48-50].

Although multiple alternative treatment strategies such as chemotherapy, biotherapy, peptide receptor radionuclide therapy (PRRT) are available for the treatment of hepatic metastases of NEN, the only option that promises a potential cure remains complete (R0) resection of the disease.

Various retrospective studies suggest that patients who undergo surgical treatment of NELM reveal a longer overall survival and more effective control of clinical symptoms [51]. Therefore, European Neuroendocrine Tumor Society (ENETS) guidelines recommend that surgery of hepatic metastases with a curative intent should always be considered in patients with a low Ki-67 index $(<20 \%)$ [52].

Even if curative surgery is impossible, debulking surgery could still be performed to alleviate symptoms, since a survival benefit has even been demonstrated, if $>80$ $90 \%$ of the tumor load was resected $[53,54]$.

The best strategy for the treatment of NELM is still poorly defined despite the existence of various guidelines and comprehensive reviews addressing this topic $[51,52]$. Thus, prospective trials evaluating the outcome and effectiveness of hepatic surgery for NELM are still missing due to the rarity and diversity of NEN. Therefore, a meticulous patient selection for hepatic surgery is very important to achieve success with even radical resections, since prediction of which patients benefit from extensive liver surgery or even debulking of NELM remains challenging due to the variable biological behavior of NEN. According to the literature and the authors' own experience, especially patients with a Ki- 67 index $<20 \%$ benefit from liver surgery of NELM by reduction of symptoms and increased survival. Patients with a Ki-67 index above $20 \%$ exhibit a very poor prognosis. Hepatic surgery should not be considered the first line treatment in this subgroup of patients and should only be performed within a multimodality or individualized concept $[51,52]$.
OLT as a therapy for patients with NELM is still controversial mainly due to the lack of long-term results and prospective studies. However, some authors have shown the same or improved survival after OLT compared to other treatment modalities [55]. Again, patient selection is pivotal to achieve good results. Therefore, the ENETS recommends OLT only after a 6 -month observation phase to better select patients with a favorable course of disease [52].

In summary, for NEN with a Ki67-index $<20 \%$ surgical resection remains the standard treatment for resectable NELM, and radical resection should be contemplated whenever possible. In addition, surgical debulking of NELM to reduce clinical symptoms should also be considered whenever a tumor clearance of $80-90 \%$ appears feasible. Liver transplantation is possible in patients with NELM, but it is only applicable in a highly selected group of patients.

\section{Liver Metastases from Noncolorectal Nonendocrine Cancer}

Despite the above mentioned success of liver surgery for other entities, resection of liver metastases of tumors other than CRC or endocrine tumors has not yet gained the same acceptance. In many cases, CRLM seem to be a "localized" disease. The reason for this phenomenon is unknown but maybe the predominant portal venous route of metastatic spread. On the other hand, other gastrointestinal tumors share the same route of metastasis (e.g., gastric or pancreatic cancer) but they have a different metastatic pattern. In these tumor entities, liver metastases are predominantly regarded as a "systemic" disease. Consequently, patients with noncolorectal nonendocrine cancer (NCRNNE) liver metastases are less likely to be referred for surgery.

From the technical point of view, liver resections for non-CRLM do not differ from resection of CRLM. Therefore, all of the surgical concepts outlined above also apply to the latter group of metastases. However, due to differences in the biology and the often more aggressive nature of the respective diseases, such concepts are less frequently used. A large, multicenter analysis of more than 1,000 liver resections for NCRNNE liver metastases changed the general attitude toward NCRNNE liver metastases and revealed that also a significant proportion of those patients benefit from surgery [56]; the analysis revealed several prognostic factors which predict the outcome after liver surgery very well and are extremely helpful for patient selection. Entities with a favorable outcome are breast, ampullary, and genitourinary cancers, while upper gastrointestinal cancers belong to the group with the worst prognosis. Moreover, patient age, the disease-free interval between the primary tumor and the diagnosis of metastasis as well as the extent of required liver surgery were predictive and were summarized into a score that 
stratifies patients into 3 risk groups preoperatively [56]. These results have thereafter been confirmed by several analyses [52]. Moreover, the development of potent systemic chemotherapy has also changed the attitude towards surgery for liver metastases of NCRNNE liver metastases (e.g., pancreatic or gastric cancer).

The evidence is increasing, e.g., that patients with "oligo-metastatic" pancreatic cancer benefit from liver resections for metachronous as well as combined resections of the pancreas and liver for synchronous liver metastases after response to neoadjuvant chemotherapy $[57,58]$.

As for the other entities, liver surgery for NCNNE liver metastases may be curative for some patients and at least may help pause systemic therapy for many patients. Therefore, liver surgery should be weighed against the expected morbidity of surgery and considered in all cases in which the balance appears acceptable.

\section{Surgical Procedures}

As outlined above, the selection of the procedure is essential for the long-term prognosis of the patient. While a formal hemihepatectomy is by far easier than, e.g., a dorso-lateral resection for tumors in segment $6 / 7$ or an antero-lateral resection for tumors in segment $5 / 8$, the preservation of parenchyma and particularly of vasculature may enable repeated liver resections in case of recurrent metastasis [59].

Parenchyma-sparing resections are associated with a higher intraoperative blood loss and an increased frequency of bile leaks, whereas major liver resections pose an increased risk of postoperative liver dysfunction. While bile leaks mainly require conservative measures or can be managed with interventional stenting or percutaneous drainage, there is neither a causal treatment nor even a bridging therapy for postoperative liver failure. Therefore, the policy of most centers for liver surgery is to accept a higher risk of comparably harmless complications while avoiding potentially life-threatening morbidities such as liver failure.

\section{Minimally Invasive Liver Surgery}

Like for colorectal or other primaries, minimal-invasive procedures trigger increasing interest. Many liver resections are also feasible through a minimal-invasive approach. A randomized trial from Norway demonstrated a lower complication rate for minimally invasive parenchyma-preserving liver resections. As indicated already in many papers in the literature before, the Norwegian study revealed a lower blood loss and a shorter hospital stay in the minimally invasive resection group. Moreover, the quality of life was significantly better during the 17 week follow-up [60].
These advantages may be the reason for an earlier postoperative oncological treatment; patients with CRLM, who underwent laparoscopic liver surgery started their postoperative chemotherapy significantly earlier than after open liver surgery [61].

For primary liver tumors in cirrhosis, minimally invasive liver surgery may have the advantage of less impairment of liver function and less damage of venous collaterals due to the smaller access through the abdominal wall. By this, decompensations of liver function or preexisting portal hypertension should occur less frequently using the minimally invasive approach. Accordingly, a very recent matched-pair analysis of 282 versus 282 patients revealed less ascites production after laparoscopic compared to open liver surgery in patients with liver cirrhosis. Moreover, patients had shorter intensive care unit and in-hospital stays after laparoscopic surgery [62].

Robotic liver surgery offers some additional advantages to laparoscopic surgery. In addition to 3-dimensional visualization of the operating field and a higher dexterity, ergonomics for the surgeon appear better than for laparoscopic surgery [63]. Moreover, some surgeons claim improved skills for vascular and biliary reconstructions. Up to now, however, a scientific comparison of robotic and laparoscopic surgery is lacking.

Therefore, both technologies should be further evaluated in comparison to open surgery and to each other.

\section{Conclusions}

Liver surgery has become a standard component of most treatment concepts for primary liver tumors and CRLM. Since morbidity and mortality have substantially decreased during recent decades, the current developments focus on the optimal surgical procedures and patient selection. While surgery used to be performed with a curative intent only, indications are currently changing. Due to the low morbidity and mortality rates in liver surgery for metastasis, liver surgery is increasingly used to pause systemic treatment and associated toxicity in case of localized disease and save treatment options for tumor recurrence.

Even if considered curative, surgery is nowadays frequently combined with chemotherapy in order to treat micrometastatic disease and to select patients with favorable diseases. The combination of both local and systemic treatment increases tumor control and might improve the quality of life of the patients.

As outlined in this article, the current developments focus on the ideal patient selection for liver surgery, as it has become a safe and effective treatment option for primary and secondary liver tumors. 


\section{Conflict of Interest Statement}

The authors declare no conflict of interests.

\section{Funding Sources}

The authors have not received funding relevant to this paper.

\section{Author Contributions}

All of the authors substantially contributed to the writing, drafting, and editing of this paper. In particular, S.H. and H.L. wrote the sections on liver cancer and colorectal metastases, while F.W. drafted the sections on endocrine and nonendocrine metastases.

\section{References}

1 Tan Y, Zhang W, Jiang L, Yang J, Yan L. Efficacy and safety of anatomic resection versus nonanatomic resection in patients with hepatocellular carcinoma: A systemic review and meta-analysis. PLoS One. 2017;12(10): e0186930.

2 Kakar S, Burgart LJ, Batts KP, Garcia J, Jain D, Ferrell LD. Clinicopathologic features and survival in fibrolamellar carcinoma: comparison with conventional hepatocellular carcinoma with and without cirrhosis. Mod Pathol. 2005 Nov; 18(11):1417-23.

3 Stipa F, Yoon SS, Liau KH, Fong Y, Jarnagin WR, D'Angelica M, et al. Outcome of patients with fibrolamellar hepatocellular carcinoma. Cancer. 2006 Mar;106(6):1331-8

4 Amini N, Ejaz A, Spolverato G, Maithel SK, Kim Y, Pawlik TM. Management of lymph nodes during resection of hepatocellular carcinoma and intrahepatic cholangiocarcinoma: a systematic review. J Gastrointest Surg. 2014 Dec;18(12):2136-48.

5 Mazzaferro V, Regalia E, Doci R, Andreola S, Pulvirenti A, Bozzetti F, et al. Liver transplantation for the treatment of small hepatocellular carcinomas in patients with cirrhosis. $\mathrm{N}$ Engl J Med. 1996 Mar;334(11):693-9.

6 Mazzaferro V, Llovet JM, Miceli R, Bhoori S, Schiavo M, Mariani L, et al.; Metroticket Investigator Study Group. Predicting survival after liver transplantation in patients with hepatocellular carcinoma beyond the Milan criteria: a retrospective, exploratory analysis. Lancet Oncol. 2009 Jan;10(1):35-43.

7 Renne SL, Woo HY, Allegra S, Rudini N, Yano H, Donadon M, et al. VETC (vessels encapsulating tumor clusters) is a powerful predictor of aggressive hepatocellular carcinoma. Hepatology. 2020 Jan;71(1):183-95.

8 Galle PR, Forner A, Llovet JM, Mazzaferro V, Piscaglia F, Raoul JL, et al.; European Association for the Study of the Liver. EASL clinical practice guidelines: management of hepatocellular carcinoma. J Hepatol. 2018 Jul; 69(1):182-236

9 Cherqui D, Laurent A, Mocellin N, Tayar C, Luciani A, Van Nhieu JT, et al. Liver resection for transplantable hepatocellular carcinoma: long-term survival and role of secondary liver transplantation. Ann Surg. 2009 Nov;250(5): 738-46.

10 Bhangui P, Allard MA, Vibert E, Cherqui D, Pelletier G, Cunha AS, et al. Salvage Versus Primary Liver Transplantation for Early Hepatocellular Carcinoma: Do Both Strategies Yield Similar Outcomes? Ann Surg. 2016 Jul 264(1):155-63
11 Menahem B, Lubrano J, Duvoux C, Mulliri A, Alves A, Costentin C, et al. Liver transplantation versus liver resection for hepatocellular carcinoma in intention to treat: an attempt to perform an ideal meta-analysis. Liver Transpl. 2017 Jun;23(6):836-44.

12 Lesurtel M, Clavien PA. international consensus conference on liver transplantation for hepatocellular carcinoma. Liver Transpl. 2010; 2011(Jun):7.

13 Chapman WC, Majella Doyle MB, Stuart JE, Vachharajani N, Crippin JS, Anderson CD, et al. Outcomes of neoadjuvant transarterial chemoembolization to downstage hepatocellular carcinoma before liver transplantation. Ann Surg. 2008 Oct;248(4):617-25.

14 Otto G, Schuchmann M, Hoppe-Lotichius M, Heise M, Weinmann A, Hansen T, et al. How to decide about liver transplantation in patients with hepatocellular carcinoma: size and number of lesions or response to TACE? J Hepatol. 2013 Aug;59(2):279-84.

15 Bartsch F, Hahn KA, Müller L, Baumgart J, Hoppe-Lotichius M, Klöckner R, et al. Intrahepatic cholangiocarcinoma: introducing the preoperative prediction score based on preoperative imaging. Hepatobiliary Pancreat Dis Int.2020.doi: 10.1016/j.hbpd.2020.08.002. 16 Shroff RT, Kennedy EB, Bachini M, BekaiiSaab T, Crane C, Edeline J, et al. Adjuvant Therapy for Resected Biliary Tract Cancer: ASCO Clinical Practice Guideline. J Clin Oncol. 2019 Apr;37(12):1015-27.

17 Folprecht G, Gruenberger T, Bechstein WO, Raab HR, Lordick F, Hartmann JT, et al. Tumour response and secondary resectability of colorectal liver metastases following neoadjuvant chemotherapy with cetuximab: the CELIM randomised phase 2 trial. Lancet Oncol. 2010 Jan;11(1):38-47.

18 Karoui M, Penna C, Amin-Hashem M, Mitry E, Benoist S, Franc B, et al. Influence of preoperative chemotherapy on the risk of major hepatectomy for colorectal liver metastases. Ann Surg. 2006 Jan;243(1):1-7.

19 Adam R, Pascal G, Azoulay D, Tanaka K, Castaing D, Bismuth $\mathrm{H}$. Liver resection for colorectal metastases: the third hepatectomy. Ann Surg. 2003 Dec;238(6):871-83.

20 Heinrich S, Lang H. Liver metastases from colorectal cancer: technique of liver resection. J Surg Oncol. 2013 May;107(6):579-84.

21 Hwang S, Lee SG, Ko GY, Kim BS, Sung KB, $\mathrm{Kim} \mathrm{MH}$, et al. Sequential preoperative ipsilateral hepatic vein embolization after portal vein embolization to induce further liver regeneration in patients with hepatobiliary malignancy. Ann Surg. 2009 Apr;249(4):608-16.
22 Lang H, de Santibañes E, Schlitt HJ, Malagó M, van Gulik T, Machado MA, et al. 10th Anniversary of ALPPS-Lessons Learned and quo Vadis. Ann Surg. 2019 Jan;269(1):114-9.

23 Eshmuminov D, Tschuor C, Raptis DA, Boss A, Wurnig MC, Sergeant G, et al. Rapid liver volume increase induced by associating liver partition with portal vein ligation for staged hepatectomy (ALPPS): is it edema, steatosis, or true proliferation? Surgery. 2017 Jun; 161(6):1549-52.

24 Linecker M, Björnsson B, Stavrou GA, Oldhafer KJ, Lurje G, Neumann U, et al. Risk Adjustment in ALPPS Is Associated With a Dramatic Decrease in Early Mortality and Morbidity. Ann Surg. 2017 Nov;266(5):779-86.

25 Kambakamba P, Linecker M, Schneider M, Reiner CS, Nguyen-Kim TD, Limani P, et al. Impact of associating liver partition and portal vein ligation for staged hepatectomy (ALPPS) on growth of colorectal liver metastases. Surgery. 2018 Feb;163(2):311-7.

26 Hasselgren K, Røsok BI, Larsen PN, Sparrelid E, Lindell G, Schultz NA, et al. ALPPS Improves Survival Compared With TSH in Patients Affected of CRLM: Survival Analysis From the Randomized Controlled Trial LIGRO. Ann Surg. 2019 Nov;Publish Ahead of Print. https://doi.org/10.1097/SLA. 0000000000003701.

27 Baumgart J, Jungmann F, Bartsch F, Kloth M, Mittler J, Heinrich S, et al. Two-Stage Hepatectomy and ALPPS for Advanced Bilateral Liver Metastases: a Tailored Approach Balancing Risk and Outcome. J Gastrointest Surg. 2019 Dec;23(12):2391-400.

28 Moris D, Ronnekleiv-Kelly S, Rahnemai-Azar AA, Felekouras E, Dillhoff M, Schmidt C, et al. Parenchymal-Sparing Versus Anatomic Liver Resection for Colorectal Liver Metastases: a Systematic Review. J Gastrointest Surg. 2017 Jun;21(6):1076-85.

29 Memeo R, de Blasi V, Adam R, Goéré D, Piardi T, Lermite E, et al.; French Colorectal Liver Metastases Working Group, Association Française de Chirurgie (AFC). Margin Status is Still an Important Prognostic Factor in Hepatectomies for Colorectal Liver Metastases: A Propensity Score Matching Analysis. World J Surg. 2018 Mar;42(3):892-901.

30 Viganò L, Procopio F, Cimino MM, Donadon M, Gatti A, Costa G, et al. Is Tumor Detachment from Vascular Structures Equivalent to R0 Resection in Surgery for Colorectal Liver Metastases? An Observational Cohort. Ann Surg Oncol. 2016 Apr;23(4):1352-60. 
31 Brudvik KW, Mise Y, Chung MH, Chun YS, Kopetz SE, Passot G, et al. RAS Mutation Predicts Positive Resection Margins and Narrower Resection Margins in Patients Undergoing Resection of Colorectal Liver Metastases. Ann Surg Oncol. 2016 Aug;23(8):2635-43.

32 Margonis GA, Sasaki K, Andreatos N, Kim Y, Merath K, Wagner D, et al. KRAS Mutation Status Dictates Optimal Surgical Margin Width in Patients Undergoing Resection of Colorectal Liver Metastases. Ann Surg Oncol. 2017 Jan;24(1):264-71.

33 Joechle K, Vreeland TJ, Vega EA, Okuno M, Newhook TE, Panettieri E, et al. Anatomic Resection Is Not Required for Colorectal Liver Metastases with RAS Mutation. J Gastrointest Surg. 2020 May;24(5):1033-9.

34 Gorgen A, Muaddi H, Zhang W, McGilvray I, Gallinger S, Sapisochin G. The new era of transplant oncology: liver transplantation for nonresectable colorectal cancer liver metastases. Can J Gastroenterol Hepatol. 2018 Jan; 2018:9531925.

35 Dueland S, Guren TK, Hagness M, Glimelius B, Line PD, Pfeiffer P, et al. Chemotherapy or liver transplantation for nonresectable liver metastases from colorectal cancer? Ann Surg. 2015 May;261(5):956-60

36 Dueland S, Syversveen T, Solheim JM, Solberg S, Grut H, Bjørnbeth BA, et al. Survival Following Liver Transplantation for Patients With Nonresectable Liver-only Colorectal Metastases. Ann Surg. 2020 Feb;271(2):2128.

37 Dueland S, Grut H, Syversveen T, Hagness M, Line PD. Selection criteria related to longterm survival following liver transplantation for colorectal liver metastasis. Am J Transplant. 2020 Feb;20(2):530-7.

38 Smedman TM, Line PD, Hagness M, Syversveen T, Grut H, Dueland S. Liver transplantation for unresectable colorectal liver metastases in patients and donors with extended criteria (SECA-II arm D study). BJS Open. 2020 Jun;4(3):467-77.

39 Dueland S, Line PD, Hagness M, Foss A, Andersen MH. Long-term quality of life after liver transplantation for non-resectable colorectal metastases confined to the liver. BJS Open. 2018 Oct;3(2):180-5.

40 Rees M, Tekkis PP, Welsh FK, O'Rourke T, John TG. Evaluation of long-term survival after hepatic resection for metastatic colorectal cancer: a multifactorial model of 929 patients. Ann Surg. 2008 Jan;247(1):125-35.
41 Margonis GA, Amini N, Buettner S, Kim Y, Wang J, Andreatos N, et al. The prognostic impact of primary tumor site differs according to the KRAS mutational status: a study by the International Genetic Consortium for Colorectal Liver Metastasis. Ann Surg. 2009. doi: 10.1097/SLA.0000000000003504.

42 Yamashita S, Chun YS, Kopetz SE, Vauthey JN. Biomarkers in colorectal liver metastases. Br J Surg. 2018 May; 105(6):618-27.

43 Lang H, Baumgart J, Heinrich S, Tripke V, Passalaqua M, Maderer A, et al. Extended Molecular Profiling Improves Stratification and Prediction of Survival After Resection of Colorectal Liver Metastases. Ann Surg. 2019 Nov;270(5):799-805.

44 Blazer DG 3rd, Kishi Y, Maru DM, Kopetz S, Chun YS, Overman MJ, et al. Pathologic response to preoperative chemotherapy: a new outcome end point after resection of hepatic colorectal metastases. J Clin Oncol. 2008 Nov; 26(33):5344-51.

45 Adam R, Delvart V, Pascal G, Valeanu A, Castaing D, Azoulay D, et al. Rescue surgery for unresectable colorectal liver metastases downstaged by chemotherapy: a model to predict long-term survival. Ann Surg. 2004 Oct;240(4):644-57.

46 Adam R, Wicherts DA, de Haas RJ, Ciacio O, Lévi F, Paule B, et al. Patients with initially unresectable colorectal liver metastases: is there a possibility of cure? J Clin Oncol. 2009 Apr;27(11):1829-35.

47 Adam R, Pascal G, Castaing D, Azoulay D, Delvart V, Paule B, et al. Tumor progression while on chemotherapy: a contraindication to liver resection for multiple colorectal metastases? Ann Surg. 2004 Dec;240(6):1052-61.

48 Frilling A, Sotiropoulos GC, Li J, Kornasiewicz $\mathrm{O}$, Plöckinger U. Multimodal management of neuroendocrine liver metastases. HPB (Oxford). 2010 Aug;12(6):361-79.

49 Cheung TT, Chok KS, Chan AC, Tsang S, Dai JW, Lang BH, et al. Long term survival analysis of hepatectomy for neuroendocrine tumour liver metastases. Sci World J. 2014 Jan; 2014:524045.

50 Fraenkel M, Faggiano A, Valk GD. Epidemiology of neuroendocrine tumors. Front Horm Res. 2015;44:1-23.

51 Chan MY, Ma KW, Chan A. Surgical management of neuroendocrine tumor-associated liver metastases: a review. Gland Surg. 2018 Feb;7(1):28-35.

52 Pavel M, O’Toole D, Costa F, Capdevila J, Gross D, Kianmanesh R, et al.; Vienna Consensus Conference participants. ENETS Consensus Guidelines Update for the Management of Distant Metastatic Disease of Intestinal, Pancreatic, Bronchial Neuroendocrine Neoplasms (NEN) and NEN of Unknown Primary Site. Neuroendocrinology. 2016; 103(2):172-85.
53 Que FG, Nagorney DM, Batts KP, Linz LJ, Kvols LK. Hepatic resection for metastatic neuroendocrine carcinomas. Am J Surg. 1995 Jan;169(1):36-42.

54 Sarmiento JM, Heywood G, Rubin J, Ilstrup DM, Nagorney DM, Que FG. Surgical treatment of neuroendocrine metastases to the liver: a plea for resection to increase survival. J Am Coll Surg. 2003 Jul;197(1):29-37.

55 Fan ST, Le Treut YP, Mazzaferro V, Burroughs AK, Olausson $\mathrm{M}$, Breitenstein $\mathrm{S}$, et al. Liver transplantation for neuroendocrine tumour liver metastases. HPB (Oxford). 2015 Jan;17(1):23-8.

56 Adam R, Chiche L, Aloia T, Elias D, Salmon R, Rivoire M, et al.; Association Française de Chirurgie. Hepatic resection for noncolorectal nonendocrine liver metastases: analysis of 1,452 patients and development of a prognostic model. Ann Surg. 2006 Oct;244(4):524-35.

57 Crippa S, Bittoni A, Sebastiani E, Partelli S, Zanon S, Lanese A, et al. Is there a role for surgical resection in patients with pancreatic cancer with liver metastases responding to chemotherapy? Eur J Surg Oncol. 2016 Oct; 42(10):1533-9.

58 Frigerio I, Regi P, Giardino A, Scopelliti F, Girelli R, Bassi C, et al. Downstaging in Stage IV Pancreatic Cancer: A New Population Eligible for Surgery? Ann Surg Oncol. 2017 Aug; 24(8):2397-403.

59 Torzilli G, McCormack L, Pawlik T. Parenchyma-sparing liver resections. Int J Surg. 2020 Apr;S1743-9191(20)30346-0.

60 Fretland AA, Dagenborg VJ, Bjørnelv GM, Kazaryan AM, Kristiansen R, Fagerland MW, et al. Laparoscopic Versus Open Resection for Colorectal Liver Metastases: The OSLOCOMET Randomized Controlled Trial. Ann Surg. 2018 Feb;267(2):199-207.

61 Tohme S, Goswami J, Han K, Chidi AP, Geller DA, Reddy S, et al. Minimally Invasive Resection of Colorectal Cancer Liver Metastases Leads to an Earlier Initiation of Chemotherapy Compared to Open Surgery. J Gastrointest Surg. 2015 Dec;19(12):2199-206.

62 Hobeika C, Fuks D, Cauchy F, Goumard C, Soubrane O, Gayet B, et al.; AFC-LLR-2018 study group. Impact of cirrhosis in patients undergoing laparoscopic liver resection in a nationwide multicentre survey. Br J Surg. 2020 Feb;107(3):268-77.

63 Grochola LF, Soll C, Zehnder A, Wyss R, Herzog P, Breitenstein S. Robot-assisted versus laparoscopic single-incision cholecystectomy: results of a randomized controlled trial. Surg Endosc. 2019 May;33(5):1482-90. 